\title{
Mechanism of ellipsometry monolayer oscillations during metalorganic vapor-phase epitaxy
}

\author{
Jeong-Sik Lee, ${ }^{\text {a) }}$ Shigeo Sugou, and Yasuaki Masumoto \\ Single Quantum Dot Project, ERATO, JST, Tsukuba Research Consortium, 5-9-9 Tokodai, \\ Tsukuba 300-2635, Japan
}

(Received 14 June 1999; accepted for publication 23 March 2000)

\begin{abstract}
Ellipsometry signal oscillations were clearly seen during metalorganic vapor-phase epitaxy (MOVPE) of III-V materials under high sample rotation at $1400 \mathrm{rpm}$. The ellipsometric signal oscillated at a period corresponding to $1 \mathrm{ML}$ of MOVPE growth. Oscillations correlated with the formation of islands due to nucleation on terraces during the growth of $1 \mathrm{ML}$. Different surface reconstructions between (100) terraces (As stable) and (111)A step edges (Ga stable) result in different dielectric functions. The effective medium dielectric response of rough and smooth surfaces appears to be responsible for different ellipsometry signals. Layer thickness and InGaAs composition were precisely determined in situ. Critical layer thickness and In composition for InGaAs quantum-dot formation were also evaluated directly from in situ ellipsometry data. (C) 2000 American Institute of Physics. [S0021-8979(00)00113-4]
\end{abstract}

\section{INTRODUCTION}

Real-time monitoring and control is highly desirable in growing low-dimensional structures. Reflection high-energy electron diffraction (RHEED) is used routinely for this in molecular-beam epitaxy (MBE). The diffraction pattern observed is used for different monitoring purposes, e.g., deoxidation of the substrate surface, temperature calibration, and thickness control via monolayer oscillations in island growth mode. In metalorganic vapor-phase epitaxy (MOVPE) under gas-phase conditions, however, electron-based in situ techniques cannot be used. To overcome this, MOVPE has been studied by techniques such as reflectance difference spectroscopy (RDS), ${ }^{1,2}$ surface photoabsorption (SPA), ${ }^{1,3}$ and spectroscopic ellipsometry (SE) ${ }^{4,5}$ In situ RDS and SE have been successfully used to monitor layer thickness, bulk composition, and surface reconstruction during III-V growth. We report well-defined ellipsometry monolayer oscillations during GaAs growth using a laser as the probe light. Compared to RDS and SE results, our data show a high signalto-noise ratio even under high sample rotation.

We evaluated the origin of oscillations from experimental data and explain ellipsometry monolayer oscillation using a model based on relative domain coverage and the surface reconstruction complex and/or Ga-alkyl bond surface concentration. Surface morphology is assignable by ellipsometry, and we observed quantum-dot (QD) formation onset. ${ }^{5,6}$ Using in situ monitoring of QD formation and monolayer oscillations, we determined alloy composition and critical layer thickness precisely for QD formation.

\section{EXPERIMENT}

A Faraday-modulated self-nulling ellipsometer is attached to the reactor at a $70^{\circ}$ angle of incidence to the

\footnotetext{
${ }^{a)}$ Present address: Optoelectronics and High Frequency Device Research Laboratory, NEC Corporation, 34 Miyukigaoka, Tsukuba 305-8501, Japan; electronic mail: jslee@obl.cl.nec.co.jp
}

sample. Determining maximum sensitivity for small changes in complex reflectivity of the sample requires a high-power probe light with minimum random noise. A $632.8 \mathrm{~nm}(1.96$ $\mathrm{eV}) \mathrm{He}-\mathrm{Ne}$ laser and $532 \mathrm{~nm}(2.32 \mathrm{eV})$ green-diode-pumped solid-state laser were used for the probe light because they meet the above requirements and have the energy of a surface resonance related to $\mathrm{Ga}$ dimers $(2 \mathrm{eV})$ at $T_{g} 500^{\circ} \mathrm{C}$ or the energy of a $\mathrm{Ga}-\mathrm{C}$ bond $(2.34 \mathrm{eV}) .^{4,7,8}$ The $\mathrm{He}-\mathrm{Ne}$ laser (532 nm laser) has a $4(20) \mathrm{mW}$ power output (spot size: 1 $\mathrm{mm}$ in diameter) and is linearly polarized with a polarization ratio of 500:1 (20:1). From the detector, the signal is transferred to a lock-in amplifier (time constant: $100 \mathrm{~ms}$ ). The interval between data acquisitions was $500 \mathrm{~ms}$. Noise levels during growth were typically $0.02^{\circ}$ for $\Delta$ and $0.01^{\circ}$ for $\Psi$ ( 0.001 for $\epsilon_{1}$ and 0.003 for $\epsilon_{2}$ ).

Our growth system is based on conventional Emcore Discovery $75 \mathrm{MOVPE}$ at a working pressure of $80 \mathrm{hPa}$. Using $1400 \mathrm{rpm}$ substrate rotation throughout growth ensured uniform source gas flow. Substrates were unintentionally doped GaAs and InAs (100) oriented. Triethylgallium (TEG), trimethylgallium (TMG), trimethylindium (TMI), and arsine $\left(\mathrm{AsH}_{3}\right)$ were the source materials.

Ex situ surface morphologies were studied by atomicforce microscopy (AFM) in air.

\section{RESULTS AND DISCUSSIONS}

Figure 1 shows transients in (a) real $\left(\epsilon_{1}\right)$ and (b) imaginary $\left(\epsilon_{2}\right)$ parts of pseudodielectric functions during GaAs growth with TEG supply as the varied parameter using a 532 $\mathrm{nm}$ probe light. An immediate decrease in $\epsilon_{1}$ and an increase in $\epsilon_{2}$ are seen when TEG is switched on, followed by a number of well-resolved oscillations. The amplitudes of $\epsilon_{1}$ and $\epsilon_{2}$ oscillations detected were 0.03 and 0.05 . Similar oscillations have been observed when TMI was used in InAs growth (Fig. 2). 

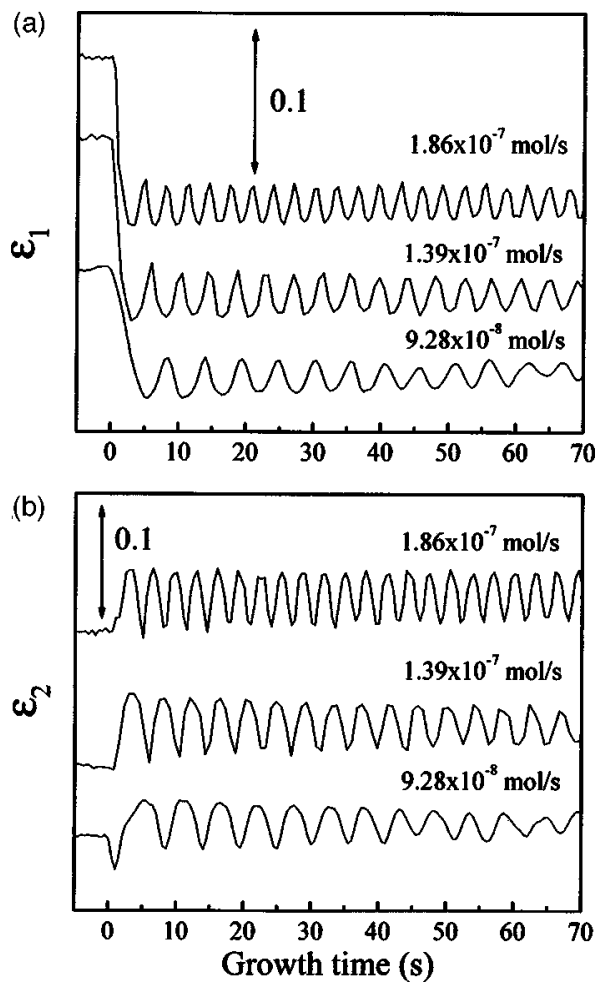

FIG. 1. Transients in (a) real $\left(\epsilon_{1}\right)$ and (b) imaginary $\left(\epsilon_{2}\right)$ parts of pseudodielectric functions during $\mathrm{GaAs}$ growth at $500^{\circ} \mathrm{C}$.

The time period of oscillations decreases with increasing source gas supply independent of the material, indicating that the time period corresponds to $1 \mathrm{ML}$ of growth. To verify this, we evaluated the growth rate by determining the layer thickness after growth (ex situ). GaAs and InAs layer thicknesses after growth were evaluated for $\operatorname{In}_{0.2} \mathrm{Ga}_{0.8} \mathrm{As}$ (3 ML)/ GaAs (100 ML) and $\operatorname{In}_{0.8} \mathrm{Ga}_{0.2}$ As (3 ML)/InAs (100 ML) superlattices by $\mathrm{x}$-ray diffraction (XRD). With the XRD system, it is possible to measure the percentage composition and layer thickness to with in $1 \%$ using routine measurements. Growth rate data are plotted in Fig. 3 together with that obtained assuming that oscillations describe the completion of a monolayer of GaAs and InAs (in situ). Linear dependence on the source gas supply and excellent agreement between growth rate data derived from the oscillation period and that derived from thickness were obtained. The deviation between in situ and ex situ data was less than $1 \%$. We thus concluded that ellipsometry in MOVPE growth has a potential equivalent to RHEED for MBE growth ${ }^{9,10}$ in characterizing the surface before growth and calibrating growth on a monolayer scale.

The mechanism generating ellipsometry oscillation is clearly cooperative, involving oscillating step density. Ellipsometry oscillations vanish when the growth mode changes from two-dimensional (2D) islands to a step flow (Fig. 4). $\operatorname{GaAs}(100)$ exactly oriented and $1^{\circ}$ off toward the [110] azimuth was used for the experiment. On a vicinal substrate, an ellipsometry signal showing clear oscillations at $T_{g}$ of 500 ${ }^{\circ} \mathrm{C}$ vanishes at $550{ }^{\circ} \mathrm{C}$, whereas oscillations are observed at both $T_{g}$ on an exactly oriented substrate. Surface morphology of the $550{ }^{\circ} \mathrm{C}$ grown sample on a vicinal substrate shows

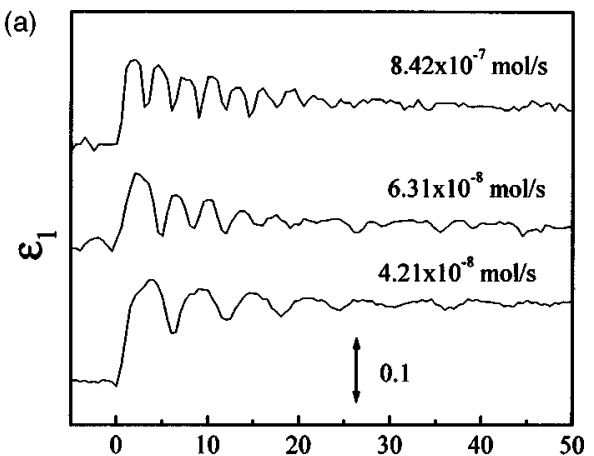

(b)

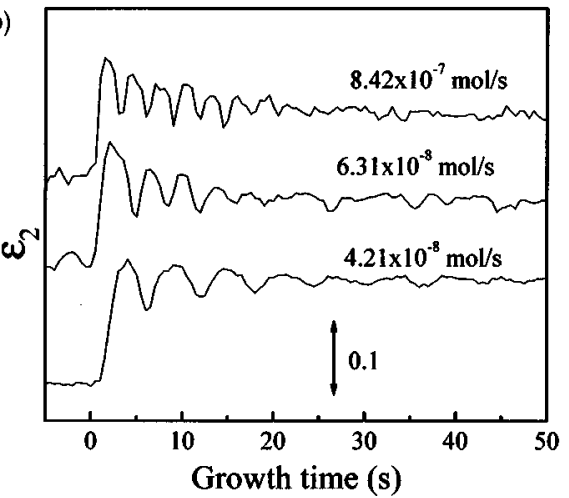

FIG. 2. Transients in (a) real ( $\left.\epsilon_{1}\right)$ and (b) imaginary $\left(\epsilon_{2}\right)$ parts of pseudodielectric functions during InAs growth at $400{ }^{\circ} \mathrm{C}$.

clear step bunching, indicating that growth proceeds in stepflow mode. The result provides additional evidence for the assumption that a condition necessary for the appearance of ellipsometry oscillations is due to oscillating step density. The result further implies that ellipsometry can also be used to determine diffusion length in MOVPE via the analysis of transition temperatures between the island growth mode to step-flow growth mode on vicinal surfaces.

The above data show a clear correlation between step density and oscillation. We studied the difference in the ellipsometric signal between terrace and step regions. Since island edges on the (100) surface consist of (111)A and B steps, the investigation of ellipsometry signal dependence on growth condition for these surfaces should help us to determine the oscillation mechanism precisely. Figure 5 shows

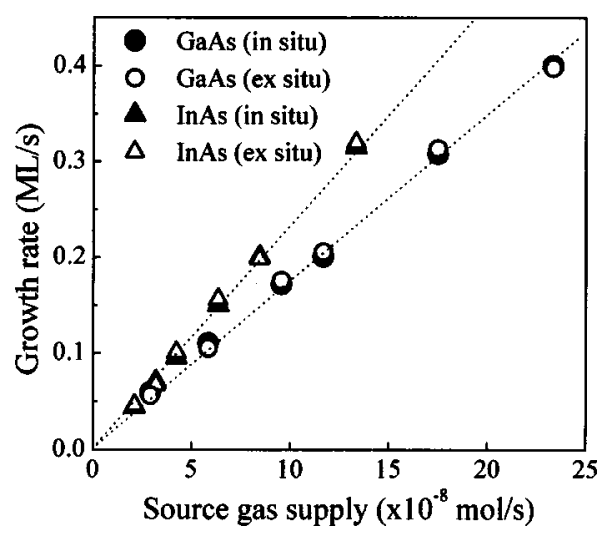

FIG. 3. GaAs and InAs growth dependence on source gas supply evaluated from growth oscillation and from postgrowth thickness determination. 


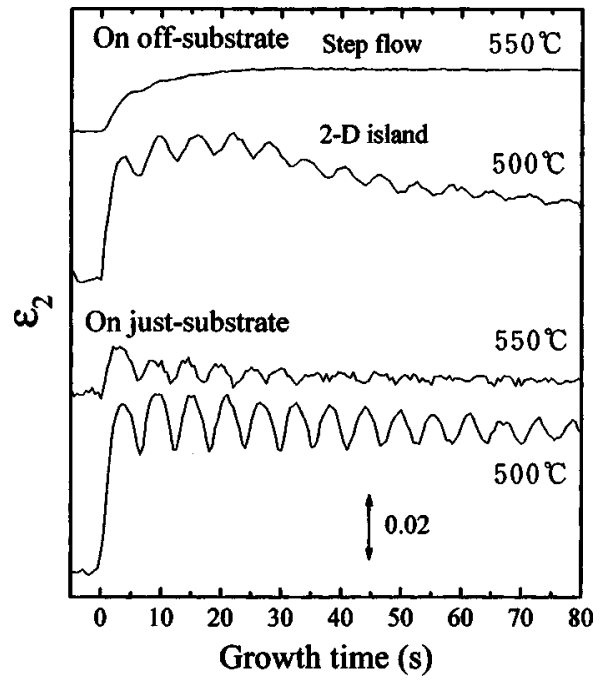

FIG. 4. Transients in imaginary parts of pseudodielectric functions $\left(\epsilon_{2}\right)$ during GaAs growth on $\mathrm{GaAs}(100)$ exactly oriented and vicinal substrates.

the temperature dependence of trajectories of the dielectric functions under $\mathrm{AsH}_{3}$ supply. At lower temperatures, no difference is seen in the trajectory between the two (111)A and B surfaces, just the same as for that of the (100) surface. At $600{ }^{\circ} \mathrm{C}$ when the temperature increases, however, the trajectory of the (111)A surface slips off, whereas that on the (111)B surface shows continuous change. With decreasing temperature, the trajectory then goes back at $560^{\circ} \mathrm{C}$. The transition is relatively fast "within a few seconds" and the transition temperature depends on the $\mathrm{AsH}_{3}$ supply. These features suggest As desorption on the (111)A surface results in a surface reconstruction change. On the (111) GaAs surface, $(2 \times 2)$ reconstruction is stable at lower temperature. The As-stabilized $(2 \times 2)$ reconstruction of the $\operatorname{GaAs}(111) \mathrm{B}$ surface consists of As trimers bonded to the underlying As plane. Surface reconstruction changes to $(\sqrt{19} \times \sqrt{19})$ at higher temperature; however, the topmost surface consists of

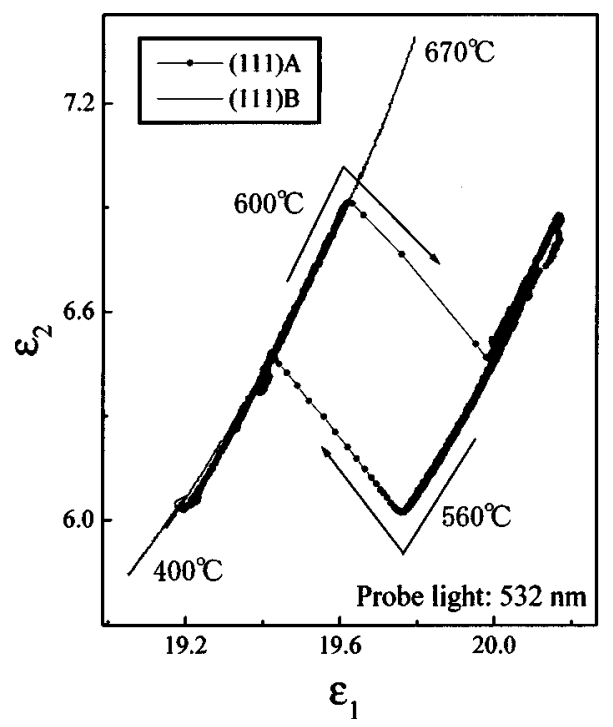

FIG. 5. Temperature dependence of trajectories of the dielectric functions under the $\mathrm{AsH}_{3}$ supply. (a) Smooth surface

\begin{tabular}{|c|c|}
\hline Ambient & $\varepsilon_{\mathrm{a}}$ \\
\hline$c(4 \times 4)$ & $\varepsilon_{\mathrm{c}(4 \times 4)}$ \\
\hline Bulk & $\varepsilon_{\mathrm{b}}$ \\
\hline
\end{tabular}

(b) Rough surface (surface reconstruction model)

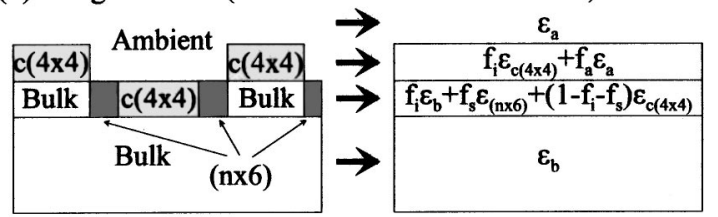

(c) Rough sourface ( $\mathrm{Ga}-\mathrm{C}$ bond model)

\begin{tabular}{|c|c|c|c|}
\hline$c\left(4 x^{4}\right)$ & Amb & $(4 \times 4)$ & $\frac{\varepsilon_{\mathrm{a}}}{\mathrm{f}_{\mathrm{i}} \varepsilon_{\mathrm{c}(4 \times 4)}+\mathrm{f}_{\mathrm{s}} \varepsilon_{\mathrm{Ga}-\mathrm{C}}+\mathrm{f}_{\mathrm{a}} \varepsilon_{\mathrm{a}}}$ \\
\hline Bulk & $c(4 \times 4)$ & Bulk & $\left(f_{i}+f_{s}\right) \varepsilon_{b}+f_{a} \varepsilon_{c(4 \times 4)}$ \\
\hline \multicolumn{3}{|c|}{ Bulk } & $\varepsilon_{\mathrm{b}}$ \\
\hline
\end{tabular}

FIG. 6. Analytical models for Bruggemann EMA. (a) Model of perfectly smooth layer. (b) Model of the layer with surface roughness with step related $(n \times 6)$-like area fraction. $f_{s}$ is covered by $c(4 \times 4)$ island area fraction $f_{i}$. (c) Model of rough surface with the $\mathrm{Ga}-\mathrm{C}$ bond at the step edges.

As. ${ }^{11}$ As-stabilized $(2 \times 2)$ reconstruction of the GaAs(111)A surface has the As trimer structure directly bonded to the Ga plane. With increasing temperature, As desorbs and $\mathrm{Ga}$, emerging on the topmost surface, forms a trimer structure. Different dielectric functions between As-As and $\mathrm{Ga}-\mathrm{Ga}$ bonds cause the change in dielectric function. The same shift was observed when the Ga source was supplied only on the (111)A surface (data not shown).

Taking the above results and step density change during $1 \mathrm{ML}$ of growth into account, we propose the elementary surface process below and calculate the origin of ellipsometry signal oscillation during GaAs growth.

In MOVPE, $c(4 \times 4)$ reconstruction is stable on the (100) GaAs surface [Fig. 6(a)]. During growth, the Ga dimer constellation is related to the (111)A step at the island edges and the $c(4 \times 4)$-like dimer constellation related to a smooth surface covers the centers of the islands [Fig. 6(b)]. Although we cannot demonstrate the surface reconstruction of the (111)A surface to the (111)A steps directly, step regions dominated by the $(n \times 6)$-like ( $n=1$ or 4$)$ structure, the mixture of As and Ga dimers, are apparently more likely to exist close to the steps. ${ }^{12,13}$

The Bruggemann effective medium approximation (EMA) (Ref. 14) has been shown to describe these effects accurately and is used here to describe oscillations in the ellipsometry response through island formation. The effective complex dielectric functions of films, obtained by EMA theory, are connected with complex dielectric functions of the GaAs surface with a mixture of reconstructions and ambient. Accordingly, the effective complex dielectric function $\epsilon$ of the GaAs topmost layer during growth is obtained from the following equations: 


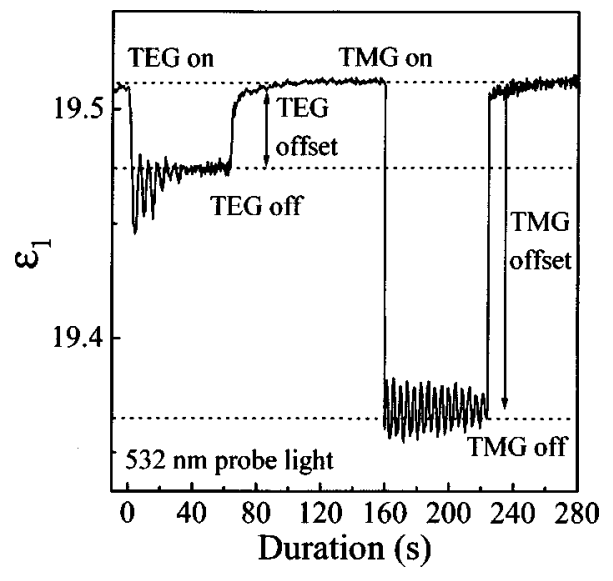

FIG. 7. Transients in the real part of the pseudodielectric function $\left(\epsilon_{1}\right)$ during GaAs growth under TEG and TMG supply.

$$
\begin{aligned}
& f_{a} \frac{\boldsymbol{\epsilon}_{a}-\boldsymbol{\epsilon}}{\boldsymbol{\epsilon}_{a}+\boldsymbol{\epsilon}}+\sum_{k=1}^{n} f_{k} \frac{\boldsymbol{\epsilon}_{k}-\boldsymbol{\epsilon}}{\boldsymbol{\epsilon}_{k}+\boldsymbol{\epsilon}}=0, \\
& f_{a}+\sum_{k=1}^{n} f_{k}=1,
\end{aligned}
$$

where $n$ is the number of distinct constituent media in the mixture, $f_{k}$ (for example, $f_{(n \times 6)}$ and $f_{c(4 \times 4)}$ ) is the volume fraction of the $k$ th component, and $\epsilon_{k}$ is the dielectric function of the $k$ th component. $f_{a}$ and $\epsilon_{a}$ correspond to the volume fraction and dielectric function of the ambient. During growth, the surface is assumed to oscillate between more $c(4 \times 4)$-like As dimers and more $(n \times 6)$-like As and $\mathrm{Ga}$ dimers. Thus, $\epsilon$ oscillates following the above equations. Smooth and rough surface models and pseudodielectric functions derived from these models during $1 \mathrm{ML}$ of growth are shown in Figs. 6(a) and 6(b). ${ }^{11}$ As in Fig. 1(b), minimum $\epsilon_{2}$ occurs for initial and final smooth surfaces and a maximum for the intermediate stage when the growing layer is about half completed. The change in $\epsilon_{2}$ showing the maximum for half-monolayer growth suggests probe light absorption by $\mathrm{Ga}$ dimer bonds at step edges because of the laser energy of a characteristic surface resonance related to Ga dimers. ${ }^{4,7}$ Since step density is highest when the layer is half grown ${ }^{11}$ and the $(n \times 6)$-like $\mathrm{Ga}$ dimer constellation dominates step regions, the density of absorbed peaks and maximum $\epsilon_{2}$ occurs at the completion of half-monolayer growth. The change in probe light absorption following step ( $\mathrm{Ga}$ dimer) density is a possible candidate for the origin of ellipsometry oscillation.

The contribution of $\mathrm{Ga}-\mathrm{C}$ bond absorption has also been suggested as the origin of the oscillation. ${ }^{15}$ Organometallic groups are likely present at the island edges since lone $\mathrm{Ga}$ pairs at the edges prevent As incorporation [Fig. 6(c)]. Figure 7 compares the $\epsilon_{1}$ offset, the difference in $\epsilon_{1}$ before and during growth, when TEG and TMG was used for Ga source materials. A different $\epsilon_{1}$ offset were observed despite no difference in the growth condition except for the Ga source. The TMG offset is three times larger than that of TEG. The same feature was observed in $\epsilon_{2}$. Further, the TEG oscillation vanishes faster than that of TMG. These results indicate that the difference in offset and oscillation correlate closely with the difference in organometallic groups. By replacing ethyl groups with methyl groups, the degree of polarity for the corresponding $\mathrm{Ga}-\mathrm{C}$ bond is strengthened due to the higher electronegativity of the methyl radical, ${ }^{3,16,17}$ preventing thermal decomposition of TMG and most of the $\mathrm{Ga}-\mathrm{C}$ bonds remain at step edges, whereas the $\mathrm{Ga}-\mathrm{C}$ bond scarcely remains in TEG. Consequently, the laser energy of $2.32 \mathrm{eV}$, near the energy of the $\mathrm{Ga}-\mathrm{C}$ bond, is expected to be sensitive enough to interfere with the bonds, and the results show the clear difference in the oscillation feature. A comparison of oscillation $T_{g}$ between TMG and TEG agrees well with the above result. ${ }^{15}$ The oscillation gradually disappears with increasing $T_{g}$, eventually vanishing. This disappearance with increasing $T_{g}$ was mainly due to the growth mode transition. The oscillations disappear for higher $T_{g}$ when nucleation occurs predominantly at step edges because the diffusion length is in the range of the average terrace width. A higher $T_{g}$ for TMG than for TEG, however, corresponds to the higher decomposition temperature of TMG and gives additional weight to the assumption that one of the origins of ellipsometry oscillations is probe light absorption by $\mathrm{Ga}-\mathrm{C}$ bonds at the step edges. In actual growth, surface reconstruction and Ga-alkyl bond-related processes would be complicated.

Precise In composition and critical layer thickness and In composition for QD formation of the InGaAs layer is obtained directly from the ellipsometry signal. Figure 8(a) shows a transient in $\epsilon_{2}$ during InGaAs growth at $500{ }^{\circ} \mathrm{C}$. The In composition changed from $0 \%(\mathrm{GaAs})$ to $50 \%$ by changing the TMI flow rate from 0 to $8.67 \times 10^{-8} \mathrm{~mol} / \mathrm{s}$. The TEG flow rate was set at $8.33 \times 10^{-8} \mathrm{~mol} / \mathrm{s}$. The oscillation period decreases with increasing TMI flow and the In composition is directly obtained by measuring the rate of increase. The In composition deduced from oscillation periods and from ex situ measurement by $\mathrm{x}$-ray diffraction agrees well [Fig. 8 (b)].

In a highly lattice-mismatched system, QD structures are self-formed due to the transition of the growth mode from 2D to three-dimensional (3D) at a certain layer thickness. This drastic change in surface morphology reflects the occurrence of inflection points in the ellipsometric trajectory. $5,6,18$ The rapid increase in $\epsilon_{2}$ [arrows in Fig. 8(a)] is caused by probe light scattering because of increased surface roughness from QD formation. Ex situ AFM measurement of $\mathrm{In}_{0.5} \mathrm{Ga}_{0.5} \mathrm{As}$ sample surfaces whose growth was stopped before and after the inflection point [indicated by arrows $\alpha$ and $\beta$ in Fig. 8(a)] clearly proved that deviation from the spiral trajectory comes from the growth mode transition [Fig. 8(c)]. QD structures were observed only on the surface after inflection points. The data show the critical point of In composition for QD formation. In our experiment, no inflection point is seen and ellipsometry oscillation remains several tens of periods when In composition is below 0.3 . The critical layer thickness of QD formation is directly calculated by dividing the oscillation period into the time period between the growth starts and the inflection occurs. The critical In composition of QD formation is estimated at 0.32 and the critical layer thickness is 14.2 ML. From Fig. 8(a), the critical layer thickness for QD formation is 5.3 (11.1) ML when In com- 


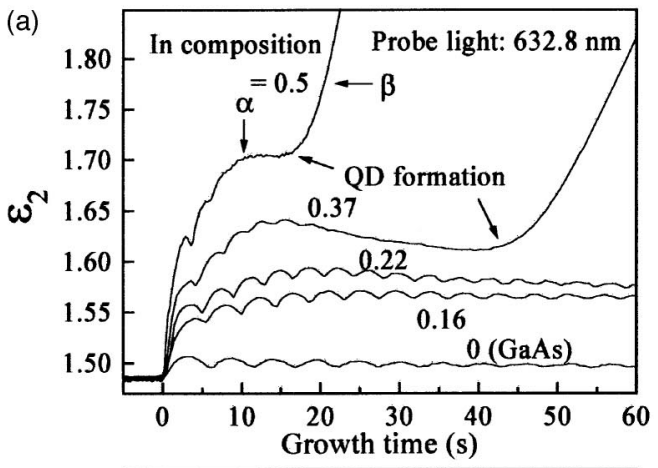

(b)

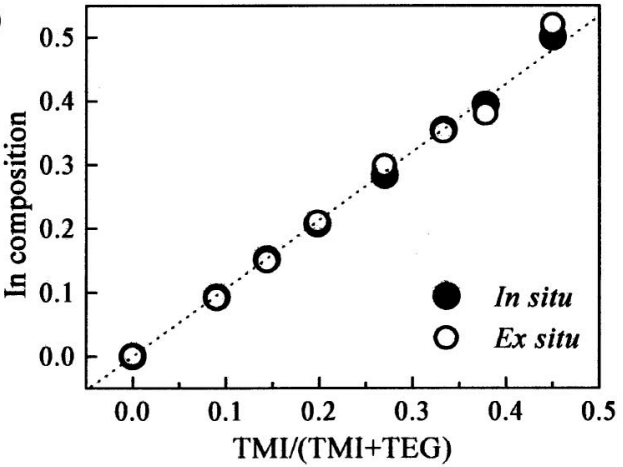

(c)

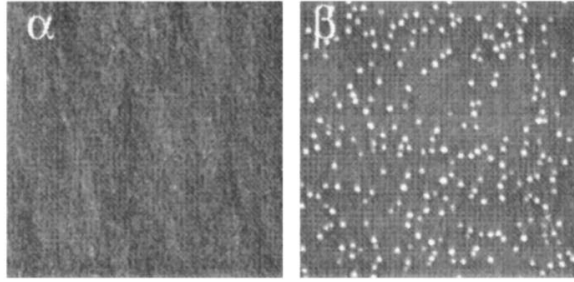

FIG. 8. Transients in (a) the imaginary $\left(\epsilon_{2}\right)$ part of pseudodielectric functions during InGaAs growth at different TMI flow rates. (b) In composition dependence on source gas evaluated from growth oscillation and from postgrowth determination. (c) AFM images of $\mathrm{In}_{0.5} \mathrm{Ga}_{0.5}$ As layers corresponding to the surface of samples for which growth was stopped at $\alpha$ and $\beta$ in Fig. 8 (a). The image area is $1 \mu \mathrm{m} \times 1 \mu \mathrm{m}$.

position is $0.5(0.37)$. Although the critical layer thickness depends on growth condition, these values match the critical layer thickness obtained from the MBE growth with RHEED oscillations under similar conditions. ${ }^{19,20}$

These results indicate that ellipsometric oscillation is a practical means for accurate in situ growth and gas flow ratio determination in MOVPE, which is important for precisely controlling lattice matching, alloy composition, quantumwell thickness, and QD formation.

\section{SUMMARY}

We observed ellipsometry oscillations during GaAs and In(Ga)As growth, and found that the ellipsometric signal oscillated at a period corresponding to $1 \mathrm{ML}$ of growth. Excellent agreement between growth rate data from the oscillation period and that from thickness measurements indicates that ellipsometry in MOVPE growth has a potential equivalent to RHEED for MBE growth in characterizing the surface before growth and calibrating the growth rate on a monolayer scale.

We determined the layer thickness and InGaAs composition precisely in situ and directly calculated the critical layer thickness and In composition for InGaAs QD formation directly from in situ ellipsometry data.

Clear ellipsometry oscillation under a high sample rotation implies that the technique enables true in situ monitoring.

${ }^{1}$ D. E. Aspnes, Surf. Sci. 307, 1017 (1994).

${ }^{2}$ I. Kamiya, D. E. Aspnes, H. Tanaka, L. T. Florez, J. P. Harbison, and R. Bhat, Phys. Rev. Lett. 68, 627 (1992).

${ }^{3}$ T. Makimoto, Y. Yamauchi, N. Kobayashi, and Y. Horikoshi, Jpn. J. Appl. Phys., Part 2 29, L645 (1990).

${ }^{4}$ D. E. Aspnes, I. Kamiya, H. Tanaka, and R. Bhat, J. Vac. Sci. Technol. B 10, 1725 (1992).

${ }^{5}$ J.-S. Lee, S. Sugou, H.-W. Ren, Y. Masumoto, and K. Kurihara, Appl. Surf. Sci. 41, 114 (1999).

${ }^{6}$ J.-S. Lee, S. Sugou, H.-W. Ren, and Y. Masumoto, J. Vac. Sci. Technol. B 17, 1341 (1999).

${ }^{7}$ M. Wassermeier, J. Behrend, J.-T. Zettler, K. Stahrenberg, and K. H. Ploog, Appl. Surf. Sci. 107, 48 (1996).

${ }^{8}$ Q. Chen and P. D. Dapkus, J. Electrochem. Soc. 138, 2821 (1991).

${ }^{9}$ J. H. Neave, B. A. Joyce, P. J. Dobson, and N. Norton, Appl. Phys. A 83, 1 (1983).

${ }^{10}$ T. Sakamoto, N. J. Kawai, T. Nakagawa, K. Ohta, and T. Kojima, Appl. Phys. Lett. 47, 617 (1985).

${ }^{11}$ D. K. Biegelsen, R. D. Bringans, J. E. Northrup, and L.-E. Swartz, Phys. Rev. Lett. 65, 452 (1990).

${ }^{12}$ K. Ploska, J.-Th. Zettler, W. Richter, J. Jonsson, F. Reinhardt, J. Rumberg, M. Pristovsek, M. Zorn, D. Westwood, and R. H. Williams, J. Cryst. Growth 145, 44 (1994).

${ }^{13}$ T. H. Chiu, W. T. Tsang, and J. E. Cunningham, J. Appl. Phys. 62, 2302 (1987).

${ }^{14}$ D. E. Aspnes, Am. J. Phys. 50, 704 (1982)

${ }^{15}$ J.-S. Lee, S. Sugou, and Y. Masumoto, Jpn. J. Appl. Phys., Part 2 38, L614 (1999).

${ }^{16}$ G. B. Stringfellow, Organometallic Vapor-Phase Epitaxy: Theory and Practice (Academic, New York, 1989).

${ }^{17}$ E. G. Rochow, D. T. Hurd, and R. N. Lewis, The Chemistry of Organometallic Compounds (Wiley, New York, 1957), p. 8.

${ }^{18}$ C. F. Bohren and D. B. Huffman, Absorption and Scattering of Light by Small Particles (Wiley, New York, 1983), p. 136.

${ }^{19}$ C. W. Snyder, J. F. Mansfield, and B. G. Orr, Phys. Rev. B 46, 9551 (1992).

${ }^{20}$ P. M. Petroff and S. P. Denbaas, Superlattices Microstruct. 15, 15 (1994). 